\title{
The Effect of Kendo's Chudan-no-kamae and Three Striking Movements on Changes in Brainwaves Related to Concentration Index
}

\author{
Yun-A Shin ${ }^{1 *}$, Sang-Min Hong ${ }^{2}$, Seung-Ho Kim³, Byung-Kun Lee ${ }^{4}$ \\ ${ }^{1}$ Department of Prescription \& Rehabilitation of Exercise, College of Physical Exercise, Dankook University, Republic of Korea \\ ${ }^{2}$ Department of Sports Rehabilitation, College of Human Care, Gimcheon University, Republic of Korea \\ ${ }^{3}$ Department of Kinesiology, College of Physical Exercise, Yong-in University, Republic of Korea \\ ${ }^{4}$ Department of Sports Physical Education, Division of Convergence of Sport, SangMyung University, Republic of Korea
}

\begin{abstract}
Received: September 26, 2020

Accepted: March 24, 2021

Published online: April 30, 2021

Keywords:

Brain Waves

Chudan-no-kamae

Kendo

Three Striking Movement

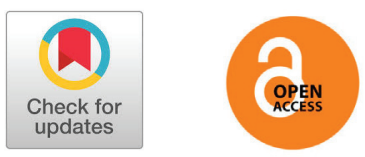

OBJECTIVES This study was conducted to figure out the effects of kendo training on the activity of brainwaves by analyzing changes in brainwaves during chudan-no-kamae and three-movement-striking, which are basic movements of kendo, in elementary school students who undergo kendo training and comparing left and right side brainwave activities to find out differences.

METHODS This study was conducted with 29 male elementary school students living in K City who were experts in kendo with a career as an athlete or a kendo training period not shorter than six months. The brainwave activity was measured by measuring and analyzing brainwaves at eight regions at the prefrontal lobe, the frontal lobe, the temporal lobe, and the occipital lobe using brainwave measuring equipment. Brainwaves were measured for 5 minutes in a sitting position in a stable state and were also measured for 5 minutes after 15 minutes of the chudan-no-kamae movement, which is aiming at the opponent with the point of the sword while moving along eight moving lines of the opponents, and for 5 minutes after 15 minutes of three striking movements.

RESULTS Alpha waves showed higher brainwave activity during the stable state than during the kendo movements (three striking movements, chudan-no-kamae) while SMR, M-beta, and H-beta showed higher brainwave activity after kendo movements than during the resting state. The asymmetry between the left and right sides increased after the movements compared to during the resting state.

CONCLUSIONS These results are considered attributable to kendo training that partly increased their concentration thereby increasing brainwave activity. However, it is unclear that kendo training will be helpful for concentration through physical activities and brain activity in this study. Therefore, further study should be conducted with the measurement of factors related to emotional aspects, concentration, and brainwaves according to kendo training.
\end{abstract}

(c) The Asian Society of Kinesiology and the Korean Academy of Kinesiology

\section{Introduction}

Physical activities are a factor that not only positively

*Correspondence: Yun-A Shin, Department of Prescription \& Rehabilitation of Exercise, College of Physical Exercise, Dankook University, San 29, Anseo-dong, Cheonan-si, Chungnam 330-714, Republic of Korea; Tel: +82-41-550-3831; Fax: +8241-550-3831; E-mail: shinagel3@gmail.com affects the cardiovascular system but also affects human information processing ability and cognitive functions [1]. Changes in the activity of the cerebral cortex according to peripheral changes are frequently measured using brainwaves in noninvasive methods [2]. Brainwaves are measured through minute electric flows on the cerebral cortex on the surface 
of the brain. In general, brainwaves are divided into delta $(0 \sim 4 \mathrm{~Hz})$, theta $(4 \sim 8 \mathrm{~Hz})$, alpha $(8 \sim 13 \mathrm{~Hz})$, beta $(13 \sim 30 \mathrm{~Hz})$, and gamma $(30 \sim 50 \mathrm{~Hz})$ waves in order of precedence beginning from the lowest frequency ending at the highest frequency. Whereas low frequencies are dominant in sleepy or resting states such as delta wave, high frequencies are dominant when performing tasks such as mental arithmetic and reasoning or when mentally aroused such as beta and gamma waves. In general, movements have been reported to increase cortical arousal and affect alpha and beta waves. Alpha waves are used as an index of states where mental or physical tension or stress is relieved $[3,4]$, and SMR waves $(12 \sim 15 \mathrm{~Hz})$ are the sensorimotor brain rhythm generated in a reticular-thalamocortical network and recorded over central scalp regions [5]. SMR waves are also used as an indicator of concentration because they are closely related to the state of readiness or attention, which corresponds to the waiting state of the motor system $[5,6]$. On the other hand, it is reported that M-beta waves, which were related to thinking activities focused on one topic and experiences brain stress and H-beta waves, which were associated with increased activity during concentration on specific tasks [7]. In addition, the concentration index (CI), which can quantitatively measure the brain activity related to concentration by using the frequency spectrum of these brain waves (theta wave, beta wave, SMR), determines the degree of concentration on one task. It increases when the attention to awakening, learning, and preparation for exercise begins [8].

Such changes in brain waves following exercise are caused by movements because movements not only increase the body's metabolism, but also affect the cortex, thereby not only having the brain to be prepared to accept new information, but also promoting learning for memory and increasing arousal and concentration for adaptation to mental changes [9]. It was reported that beta waves increased after one-time incremental load exercise and that the increase in beta waves as such persisted up to two hours after exercise [10]. Such studies suggest that changes in brain waves made through exercise mean improvement in concentration [11,12], and showed positive effects on learning and cognitive ability, so that the importance of exercise as a method of improving the brain is increasing.
Although sports and athletic contests require concentration or high levels of brain activities, especially in kendo, shooting, archery, golf, etc., concentration and brain activities greatly affect the victory or defeat or the outcomes of contests [13]. Kendo as a form of exercise is a sport that has a stronger tendency toward martial arts than general competitive sports and has a characteristic of requiring mental and physical training and the cultivation of mentality to improve athletic performance [14]. Kendo matches, in which the victory or defeat is determined by quick brain plays and agile actions, require mental stability and physical concentration. That is, since violent collisions and tense confrontations with the opponent are repeated in kendo matches, a strong fighting spirit, human talent, and concentration greatly affect the outcomes of the matches [15].

In kendo, chudan-no-kamae is a posture aiming at the opponent that enables attacks, defense, and flexible responses to the opponent's movements and is also called "basic posture" of kendo. The three striking movements, which are the basic of strikes, are movements consisting of movement 1 to raise the bamboo sword, movement 2 to take one step forward and strike the opponent, and movement 3 to return to the original posture and take the chudan-nokamae, and require concentration for flexible movements, stable postures, and accurate movements [16]. Since both the basic postures and matches of kendo require concentration as such, kendo training is thought to help elementary school students improve their concentration. However, chudan-nokamae is relatively static motion in which the foot moves to the minimum according to the opponent's movements while focusing the bamboo sword, the three striking movements is a more dynamic motion that moves the arms together while the foot moves and strikes the bamboo sword. EEG is reported to vary depending on the type of exercise (treadmill, bicycle, arm crank). As a result of measuring healthy people, alpha activity increases in the forehead lobe area after treadmill exercise, and alpha activity in the temporal lobe increases after cycling exercise. However, the activity reported no change after arm crank exercise [17]. Therefore, it is assume that the effect of the kendo movement on the brain waves is different.

Attention concentration is one of the most important 
elements of not only the improvement of children's academic achievement ability but also leading normal school life. When the concentration of a student has been impaired, his/her ability to learn is impaired and he/she cannot get along well with other children in many cases. Recently, in the field of education, there has been a growing number of children who are marginalized by their friends due to their lagging academic achievement ability and basic academic skills resulting from their lack of attention concentration, and attention to students with insufficient attention concentration has been increasing [18]. However, studies related to kendo and brain waves are insufficient because they are limited to reports on image stimulation [14] and sound wave application training [19] for kendo athletes.

Recent studies reported that the asymmetry of the frontal lobe reflects responses to movement emotions [20]. The studies reported that the right side of the frontal lobe was associated with negative emotions, and the left side was related to motivation and happiness. The increase in alpha wave activity on the right side is interpreted as a decrease in right cortical activity [21-24]. However, inconsistent findings are still reported because on the other hand, some studies reported that the amplification of alpha waves when the frontal lobe was stable reflected an overexcited state of the cortex or a decrease in cortical activity [25]. In particular, despite that kendo is an activity that requires attention concentration, there has been no report on how brainwave activity changes on the left and right sides.

Therefore, this study aims to analyze changes in brainwaves during the chudan-no-kamae movement and three striking movements, which are the basic movements of kendo in elementary school students who undergo kendo training, and compare the left and right side brainwave activities to figure out differences with a view to understanding the effect of kendo training on the activity of brainwaves.

\section{Methods}

\section{Subjects}

This study was conducted with 29 elementary school students living in K City who were experts in kendo with a career as an athlete or a kendo training period not shorter than six months. The subjects of the study were elementary school students who well-understood the purpose of the study, and had no physical defects, musculoskeletal problems, or disease being treated and their parents' consent was obtained before they participated in the study $<$ Table $1>$.

\section{Brainwave activity}

The brainwave activity was measured by measuring and analyzing brainwaves (QEEG-LEX3208, Laxtha Inc, Daejeon, Korea) at eight regions, which are at the left and right sides of the prefrontal lobe (Fp1, Fp2), the left and right sides of the frontal lobe (F3, F4), the left and right sides of the temporal lobe (T3, T4), and the left and right sides of the occipital lobe (P3, P4) using the method used by Gasser et.al. [26]. The brainwaves in a resting state were measured for 5 minutes after having the subject sit on a comfortable chair, maintain a comfortable state, close the eyes in a noise-blocked laboratory. In addition, brainwaves were also measured for 5 minutes in a sitting position after carrying out 15 minutes of the chudan-no-kamae movement of kendo, which is aiming at the opponent with the point of the sword while moving along eight moving lines of the opponent in the chudan-no-kamae that enables attacks, defense, and flexible responses to the opponent's movements and is the basic of all aiming postures having the point of the sword at the height of the larynx and the extension of the point of the sword aiming at the middle of the forehead of the opponent, and for 5 minutes after carrying out 15 minutes of three striking movements consisting of movement 1 to raise the bamboo sword, movement 2 to take one step forward and strike the opponent, and movement 3 to return to the original posture.

Table 1. Study subjects' physical characteristics. Data are expressed as means $\pm S D$

\begin{tabular}{lllllll}
\hline Variables & Age (years) & Weight $(\mathbf{k g})$ & BMI $\left(\mathbf{k g} / \mathbf{m}^{2}\right)$ & Muscle mass $(\mathbf{k g})$ & Body percent fat (\%) & Carrier (month) \\
\hline Subjects $(\mathrm{n}=29)$ & $11.96 \pm 0.94$ & $40.43 \pm 8.75$ & $19.15 \pm 3.06$ & $1855 \pm 14.19$ & $20.95 \pm 3.77$ & $11.38 \pm 5.56$ \\
\hline
\end{tabular}


The sampling frequency of the EEG signal was set to $256 \mathrm{~Hz}$, and the measured data were analyzed using the biosignal analysis software TeleScan at $4 \sim 30 \mathrm{~Hz}$ for the purpose of the study. Analysis of the collected brain waves was performed by Fast Fourier Transform (FFT) using the TeleScan program. Relative power analysis was conducted to eliminate individual differences, as the differences in brain waves may be affected by differences in skulls, differences in electrical resistance according to scalp conditions, and differences in tension at the time of measurement. EEG activity was analyzed in all 5 minutes during the resting state and after exercise. Using the analyzed brainwaves, concentration index $(\mathrm{CI}=(\mathrm{SMR}+\mathrm{MB}) /$ theta $)$ and relaxation index $(\mathrm{RI}=\mathrm{alph} / \mathrm{HB})$ were calculated [27]. The electroencephalographic electrode attachment site is shown in $<$ Figure $1>$.

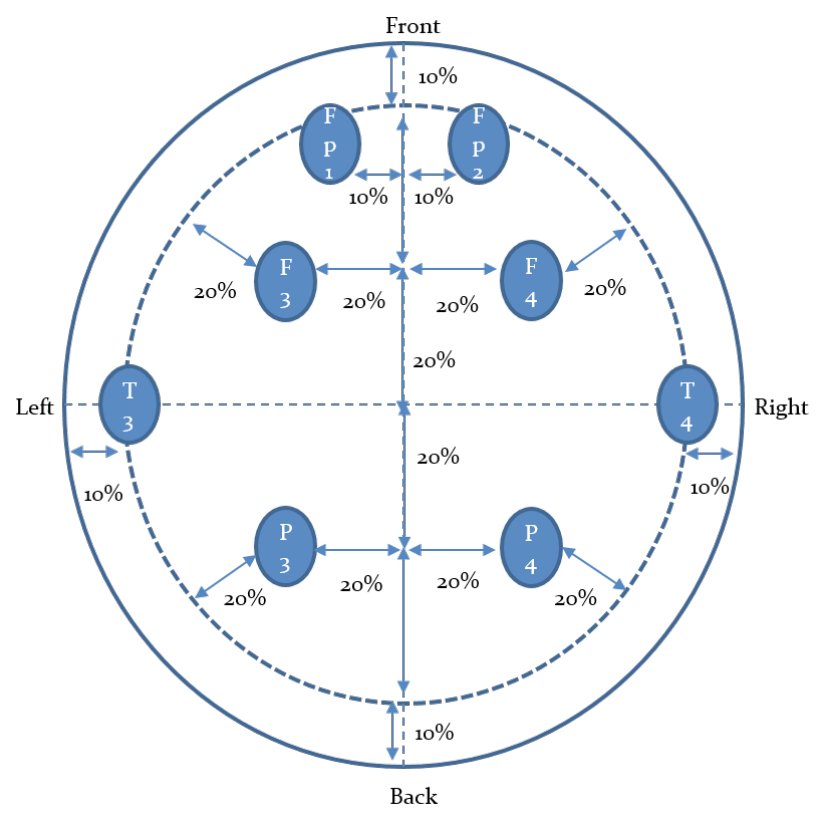

Figure 1. Electroencephalographic electrode attachment site

\section{Statistical analysis}

As for the data processing in this study, the means and standard deviations of individual variables were calculated and compared using version 20.0 of the SPSS statistical program for Windows. The brainwave activities on the left and right sides were compared using paired t-test to figure out differences and the brainwave activities during the resting state, after three striking movements, and after chudan-no- kamae movement were compared using multivariate analysis of variance (MANOVA) to figure out differences. The significance of all statistical levels was set to $\alpha=.05$.

\section{Results}

\section{Changes in alpha activity according to kendo movement}

Changes in alpha activity according to kendo movements are as shown in $<$ Table $2>$. According to kendo movements, significant differences in the alpha wave activity appeared on the left side of the frontal lobe $(\mathrm{p}<.05)$ and the left and right sides of the temporal lobe $(\mathrm{p}<.001)$. On the left side of the frontal lobe, the alpha wave activity was shown to be significantly higher during the resting state than after the chudan-no-kamae movement ( $\mathrm{p}<.01)$, and on the left and right sides of the temporal lobe, the alpha wave activity was shown to be significantly higher during the resting state than after the three striking movements and after the chudanno-kamae movement $(\mathrm{p}<.001)$. As for the differences in the alpha wave activity between the left and right sides, during the resting state, the alpha wave activity on the left side of the temporal lobe was shown to be significantly higher than that on the right side $(\mathrm{p}<.01)$ and the alpha wave activity on the left side of the occipital lobe was shown to be significantly higher than that on the right side $(\mathrm{p}<.05)$. After the three striking movements, the left side of the temporal lobe showed higher alpha wave activity than the right side $(\mathrm{p}<.01)$ and after the chudan-no-kamae movement $(\mathrm{p}<.001)$, the left side of the prefrontal lobe and the temporal lobe showed higher alpha wave activity than the right side $(\mathrm{p}<.01)$.

\section{Changes in SMR activity according to kendo movement}

Changes in SMR activity according to kendo movements are as shown in $<$ Table $3>$. According to kendo movements, significant differences in the SMR activity appeared in all brain regions. The SMR activity was shown to be significantly lower during the resting state than after the three striking movements and chudan-no-kamae movement $(\mathrm{p}<.001)$. As for the differences in the SMR activity between the left and right sides, the SMR activity on the left side of the occipital 
Table 2. Changes in alpha wave activity according to kendo movements. Data are expressed as means \pm SD

\begin{tabular}{lllllll}
\hline Variables & $\mathbf{R}$ & $\mathbf{T}$ & $\mathbf{C}$ & $\mathbf{F}$ & Sig & Post-hoc \\
\hline Fp1 & $6.71 \pm 5.03$ & $8.08 \pm 13.68$ & $3.68 \pm 3.93^{\# \# \#}$ & 1.929 & .152 & - \\
Fp2 & $22.08 \pm 9.13$ & $13.22 \pm 3.33$ & $3.31 \pm 3.79$ & .879 & .419 & - \\
F3 & $14.36 \pm 2.27$ & $7.29 \pm 10.28$ & $3.76 \pm 3.44$ & 3.998 & .022 & R C $^{* *}$ \\
F4 & $12.83 \pm 3.76$ & $9.81 \pm 2.71$ & $3.70 \pm 5.71$ & .863 & .426 & - \\
T3 & $14.64 \pm 12.17^{\# \#}$ & $5.18 \pm 7.96^{\# \#}$ & $2.93 \pm 2.50^{\# \# \#}$ & 15.407 & .000 & R>T, C*** \\
T4 & $10.98 \pm 7.16$ & $4.12 \pm 7.47$ & $2.12 \pm 2.52$ & 16.566 & .000 & R>T, C \\
P3 & $13.19 \pm 12.94^{\# * *}$ & $7.17 \pm 9.70$ & $8.64 \pm 19.58$ & 1.327 & .271 & - \\
P4 & $8.18 \pm 8.35$ & $6.06 \pm 9.46$ & $5.17 \pm 14.69$ & .557 & .575 & - \\
\hline
\end{tabular}

Fp1: prefrontal left lobe, Fp2: prefrontal right lobe, F3: frontal left lobe, F4: frontal right lobe, T3: temporal left lobe, T4: temporal right lobe, P3: occipital left lobe, P4: occipital right lobe, R: rest, T: three striking movement, C: chudan-no-kamae movement, *: the difference according to kendo movement, * $p<.05,{ }^{* *} p<.01, *$ : the difference between right and left side lobe, ${ }^{*} p<.05,{ }^{* \#} p<.01,{ }^{\# \# *} p<.001$

Table 3. Changes in SMR activity according to kendo movements. Data are expressed as means \pm SD

\begin{tabular}{|c|c|c|c|c|c|c|}
\hline Variables & $\mathbf{R}$ & $\mathbf{T}$ & C & $\mathbf{F}$ & Sig & Post-hoc \\
\hline Fp1 & $0.24 \pm 0.09$ & $0.39 \pm 0.12$ & $0.39 \pm 0.16$ & 13.364 & .000 & $\mathrm{R}<\mathrm{T}, \mathrm{C}^{* * *}$ \\
\hline $\mathrm{Fp} 2$ & $0.24 \pm 0.09$ & $0.41 \pm 0.12$ & $0.42 \pm 0.18$ & 14.837 & .000 & $\mathrm{R}<\mathrm{T}, \mathrm{C}^{* * *}$ \\
\hline F3 & $0.25 \pm 0.12$ & $0.43 \pm 0.14$ & $0.45 \pm 0.12$ & 22.302 & .000 & $\mathrm{R}<\mathrm{T}, \mathrm{C}^{* * *}$ \\
\hline F4 & $0.23 \pm 0.11$ & $0.41 \pm 0.12$ & $0.46 \pm 0.18$ & 22.458 & .000 & $R<T, C^{* * * *}$ \\
\hline T3 & $0.18 \pm 0.12$ & $0.43 \pm 0.16$ & $0.46 \pm 0.14$ & 39.949 & .000 & $\mathrm{R}<\mathrm{T}, \mathrm{C}^{* \cdots *}$ \\
\hline $\mathrm{T} 4$ & $0.18 \pm 0.12$ & $0.46 \pm 0.14$ & $0.51 \pm 0.15$ & 50.949 & .000 & $R<T, C^{* * * *}$ \\
\hline P3 & $0.31 \pm 0.19$ & $0.49 \pm 0.15^{\#}$ & $0.52 \pm 0.22$ & 10.188 & .000 & $R<T, C^{* * *}$ \\
\hline P4 & $0.37 \pm 0.21$ & $0.57 \pm 0.16$ & $0.55 \pm 0.17$ & 11.036 & .000 & $R<T, C^{* * * *}$ \\
\hline
\end{tabular}

Fp1: prefrontal left lobe, Fp2: prefrontal right lobe, F3: frontal left lobe, F4: frontal right lobe, T3: temporal left lobe, T4: temporal right lobe, P3 occipital left lobe, P4: occipital right lobe, R: rest, T: three striking movement, C: chudan-no-kamae movement, ${ }^{*}$ : the difference according to kendo movement, ${ }^{* * *}$ $p<.001,{ }^{*}:$ the difference between right and left side lobe, ${ }^{\#} p<.05$

lobe was shown to be significantly lower than that on the right side after the three striking movements $(\mathrm{p}<.05)$.

\section{Changes in MB activity according to kendo movement}

Changes in MB activity according to kendo movements are as shown in $<$ Table $4>$. Significant differences in MB activity appeared in all brain regions according to kendo movements $(\mathrm{p}<.001)$. MB activity was shown to be significantly lower during the resting state than after the three striking movements and chudan-no-kamae movement $(\mathrm{p}<.01)$. As for the differences in MB activity between the left and right sides, during the resting state, $\mathrm{MB}$ activity on the left side of the occipital lobe was shown to be lower than that of the right side $(\mathrm{p}<.05)$, and after the three striking movements, the $\mathrm{MB}$ activity on the left side of the temporal lobe $(\mathrm{p}<.01)$ and the occipital lobe $(\mathrm{p}<.001)$ was shown to be significantly lower than that on the right side. After the chudan-no-kamae movement, the MB activity on the left side of the prefrontal lobe $(\mathrm{p}<.05)$ and the temporal lobe $(\mathrm{p}<.01)$ was shown to be significantly lower than that on the right side. 
Table 4. Changes in MB activity according to kendo movements. Data are expressed as means \pm SD

\begin{tabular}{|c|c|c|c|c|c|c|}
\hline Variables & $\mathbf{R}$ & $\mathbf{T}$ & C & $\mathbf{F}$ & Sig & Post-hoc \\
\hline Fp1 & $0.33 \pm 0.18$ & $0.55 \pm 0.26$ & $0.62 \pm 0.28^{\#}$ & 11.570 & .000 & $\mathrm{R}<\mathrm{T}^{* *}, \mathrm{C}^{* * *}$ \\
\hline $\mathrm{F} 3$ & $0.34 \pm 0.21$ & $0.58 \pm 0.22$ & $0.65 \pm 0.21$ & 16.209 & .000 & $\mathrm{R}<\mathrm{T}, \mathrm{C}^{* * *}$ \\
\hline $\mathrm{F} 4$ & $0.29 \pm 0.14$ & $0.56 \pm 0.19$ & $0.62 \pm 0.22$ & 21.979 & .000 & $\mathrm{R}<\mathrm{T}, \mathrm{C}^{* * *}$ \\
\hline T3 & $0.20 \pm 0.14$ & $0.51 \pm 0.23^{\# \#}$ & $0.60 \pm 0.21^{\# \#}$ & 34.043 & .000 & $\mathrm{R}<\mathrm{T}, \mathrm{C}^{* * *}$ \\
\hline $\mathrm{T} 4$ & $0.20 \pm 0.15$ & $0.60 \pm 0.25$ & $0.67 \pm 0.22$ & 40.463 & .000 & $\mathrm{R}<\mathrm{T}, \mathrm{C}^{* * *}$ \\
\hline P3 & $0.43 \pm 0.37^{\#}$ & $0.72 \pm 0.27^{\# \# \#}$ & $0.80 \pm 0.30$ & 10.849 & .000 & $\mathrm{R}<\mathrm{T}^{* *}, \mathrm{C}^{* * *}$ \\
\hline P4 & $0.52 \pm 0.34$ & $0.85 \pm 0.33$ & $0.88 \pm 0.35$ & 9.922 & .000 & $\mathrm{R}<\mathrm{T}, \mathrm{C}^{* * *}$ \\
\hline
\end{tabular}

Fp1: prefrontal left lobe, Fp2: prefrontal right lobe, F3: frontal left lobe, F4: frontal right lobe, T3: temporal left lobe, T4: temporal right lobe, P3: occipital left lobe, P4: occipital right lobe, R: rest, T: three striking movement, C: chudan-no-kamae movement, *: the difference according to kendo movement,

${ }^{* *} p<.01,{ }^{* * *} p<.001,{ }^{\#}$ : the difference between right and left side lobe, ${ }^{\#} p<.05,{ }^{\# \#} p<.01,{ }^{\# \# \#<.001}$

Table 5. Changes in HB activity according to kendo movements. Data are expressed as means \pm SD

\begin{tabular}{|c|c|c|c|c|c|c|}
\hline Variables & $\mathbf{R}$ & $T$ & C & $\mathbf{F}$ & Sig & Post-hoc \\
\hline Fp1 & $0.65 \pm 0.62$ & $1.17 \pm 0.77$ & $1.40 \pm 0.95$ & 6.777 & .002 & $R<T^{*}, C^{* *}$ \\
\hline $\mathrm{Fp} 2$ & $0.68 \pm 0.85$ & $1.28 \pm 1.16$ & $1.54 \pm 1.26$ & 4.693 & .012 & $R<T^{*}, C^{* *}$ \\
\hline F3 & $0.49 \pm 0.34$ & $0.91 \pm 0.54$ & $0.96 \pm 0.32$ & 11.451 & .000 & $\mathrm{R}<\mathrm{T}, \mathrm{C}^{* * *}$ \\
\hline F4 & $0.48 \pm 0.33$ & $0.90 \pm 0.48$ & $1.00 \pm 0.39$ & 13.952 & .000 & $\mathrm{R}<\mathrm{T}, \mathrm{C}^{* * *}$ \\
\hline T3 & $0.29 \pm 0.29$ & $0.78 \pm 0.57^{\# \#}$ & $0.84 \pm 0.41^{\# \#}$ & 14.088 & .000 & $\mathrm{R}<\mathrm{T}, \mathrm{C}^{* * *}$ \\
\hline T4 & $0.30 \pm 0.25$ & $0.94 \pm 0.66$ & $1.01 \pm 0.52$ & 17.853 & .000 & $\mathrm{R}<\mathrm{T}, \mathrm{C}^{* * *}$ \\
\hline P3 & $0.79 \pm 0.79$ & $1.46 \pm 0.90^{\# \#}$ & $1.37 \pm 0.64$ & 6.220 & .003 & $R<T, C^{* *}$ \\
\hline P4 & $0.93 \pm 0.65$ & $1.67 \pm 0.94$ & $1.50 \pm 0.70$ & 7.186 & .001 & $R<T, C^{* *}$ \\
\hline
\end{tabular}

Fp1: prefrontal left lobe, Fp2: prefrontal right lobe, F3: frontal left lobe, F4: frontal right lobe, T3: temporal left lobe, T4: temporal right lobe, P3: occipital left lobe, P4: occipital right lobe, R: rest, T: three striking movement, C: chudan-no-kamae movement, *: the difference according to kendo movement, * $\mathrm{p}<.05,{ }^{* *} \mathrm{p}<.01,{ }^{* * *} \mathrm{p}<.001,{ }^{\#}:$ the difference between right and left side lobe, ${ }^{\# \#} \mathrm{p}<.01$

\section{Changes in HB activity according to kendo movement}

Changes in $\mathrm{HB}$ activity according to kendo movements are as shown in $<$ Table $5>$. Significant differences in $\mathrm{HB}$ activity appeared in all brain regions according to kendo movements $(\mathrm{p}<.05)$. HB activity was shown to be significantly lower during the resting state than after the three striking movements and chudan-no-kamae movement $(\mathrm{p}<.05)$. As for the differences in $\mathrm{HB}$ activity between the left and right sides, after the three striking movements, $\mathrm{HB}$ activity on the left side of the temporal lobe and the occipital lobe was shown to be lower than that on the right side $(\mathrm{p}<.01)$, and after the chudan-no- kamae movement, HB activity on the left side of the occipital lobe was shown to be lower than that on the right side $(\mathrm{p}<.01)$.

\section{Changes in concentration index according to kendo movement}

Changes in CI according to kendo movements are as shown in $<$ Table $6>$. Significant differences in CI appeared in temporal and occipital regions according to kendo movements $(\mathrm{p}<.05)$. CI was shown to be significantly lower during the resting state than after the three striking movements $(\mathrm{p}<.05)$. As for the differences in CI between the left and right sides, 
Table 6. Changes in concentration index according to kendo movements. Data are expressed as means \pm SD

\begin{tabular}{|c|c|c|c|c|c|c|}
\hline Variables & $\mathbf{R}$ & $\mathbf{T}$ & C & $\mathbf{F}$ & Sig & Post-hoc \\
\hline Fp1 & $0.30 \pm 0.27$ & $0.41 \pm 0.23$ & $0.36 \pm 0.28$ & 1.297 & .279 & - \\
\hline Fp2 & $0.31 \pm 0.29$ & $0.45 \pm 0.31$ & $0.39 \pm 0.31$ & 1.544 & .219 & - \\
\hline F3 & $0.64 \pm 0.42$ & $0.72 \pm 0.50$ & $0.63 \pm 0.27$ & .497 & .610 & - \\
\hline F4 & $0.58 \pm 0.24$ & $0.70 \pm 0.54$ & $0.65 \pm 0.20$ & .853 & .430 & - \\
\hline T3 & $0.67 \pm 0.30$ & $0.89 \pm 0.80$ & $0.73 \pm 0.31^{\#}$ & 1.396 & .253 & - \\
\hline T4 & $0.65 \pm 0.27$ & $1.02 \pm 0.92$ & $0.82 \pm 0.28$ & 3.007 & .055 & $\mathrm{R}<\mathrm{T}^{*}$ \\
\hline P3 & $1.15 \pm 0.46$ & $1.48 \pm 1.22^{\# \#}$ & $1.48 \pm 1.07$ & 1.118 & .332 & - \\
\hline P4 & $1.31 \pm 1.33$ & $1.87 \pm 1.02$ & $1.28 \pm 0.53$ & 3.022 & .054 & $\mathrm{~T}>\mathrm{R}, \mathrm{C}^{*}$ \\
\hline
\end{tabular}

Fp1: prefrontal left lobe, Fp2: prefrontal right lobe, F3: frontal left lobe, F4: frontal right lobe, T3: temporal left lobe, T4: temporal right lobe, P3: occipital left lobe, P4: occipital right lobe, R: rest, T: three striking movement, C: chudan-no-kamae movement, *: the difference according to kendo movement, * $p<.05, "$ : the difference between right and left side lobe, ${ }^{\#} p<.05,{ }^{\# \#} p<.01$

Table 7. Changes in relaxation index according to kendo movements. Data are expressed as means \pm SD

\begin{tabular}{|c|c|c|c|c|c|c|}
\hline Variables & $\mathbf{R}$ & $\mathbf{T}$ & C & $\mathbf{F}$ & Sig & Post-hoc \\
\hline Fp1 & $10.32 \pm 2.79$ & $6.91 \pm 3.48$ & $2.62 \pm 4.71$ & 2.543 & .085 & - \\
\hline Fp2 & $32.47 \pm 1.18$ & $10.33 \pm 6.06$ & $2.15 \pm 4.19$ & 1.017 & .356 & - \\
\hline F3 & $29.31 \pm 6.06$ & $8.01 \pm 2.47$ & $3.91 \pm 3.61$ & 7.289 & .001 & $\mathrm{R}>\mathrm{T}^{* *}, \mathrm{C}^{* * *}$ \\
\hline F4 & $26.73 \pm 22.31$ & $10.90 \pm 7.74$ & $3.70 \pm 6.09$ & 1.267 & .287 & - \\
\hline T3 & $50.48 \pm 30.34$ & $6.64 \pm 2.25$ & $3.49 \pm 3.31$ & 7.229 & .001 & $\mathrm{R}>\mathrm{T}, \mathrm{C}^{* *}$ \\
\hline T4 & $36.60 \pm 16.81$ & $4.38 \pm 1.83$ & $2.10 \pm 2.02$ & 8.135 & .001 & $\mathrm{R}>\mathrm{T}, \mathrm{C}^{* *}$ \\
\hline P3 & $16.79 \pm 12.31^{\# \#}$ & $4.91 \pm 2.69$ & $6.31 \pm 16.57$ & 1.329 & .270 & - \\
\hline P4 & $8.80 \pm 7.06$ & $3.63 \pm 19.15$ & $3.45 \pm 13.01$ & 1.647 & .199 & - \\
\hline
\end{tabular}

Fp1: prefrontal left lobe, Fp2: prefrontal right lobe, F3: frontal left lobe, F4: frontal right lobe, T3: temporal left lobe, T4: temporal right lobe, P3: occipital left lobe, P4: occipital right lobe, R: rest, T: three striking movement, C: chudan-no-kamae movement, *: the difference according to kendo movement, ** $\mathrm{p}<.01,{ }^{* * *} \mathrm{p}<.001,{ }^{\#}$ : the difference between right and left side lobe, ${ }^{\# \#} \mathrm{p}<.01$

after the three striking movements, CI on the left side of the occipital lobe was shown to be lower than that on the right side $(\mathrm{p}<.01)$, and after the chudan-no-kamae movement, CI on the left side of the temporal lobe was shown to be lower than that on the right side $(\mathrm{p}<.05)$.

\section{Changes in relaxation index according to kendo movement}

Changes in RI according to kendo movements are as shown in $<$ Table $7>$. Significant differences in RI appeared in frontal, temporal and occipital regions according to kendo movements $(\mathrm{p}<.05)$. RI was shown to be significantly higher during the resting state than after the three striking movements and after the chudan-no-kamae movement $(\mathrm{p}<.01)$. As for the differences in RI between the left and right sides, during the resting state, RI on the left side of the occipital lobe was shown to be higher than that on the right side $(\mathrm{p}<.01)$.

\section{Discussion}

According to the results of this study, alpha waves showed the highest activity during the resting state, and SMR, MB, and 
$\mathrm{HB}$ activities showed higher activity during the three striking movements and chudan-no-kamae movement than during the resting state. As for the differences in brainwave activity between the left and right sides, the degree of asymmetry was shown to be high in the temporal lobe and the occipital lobe during kendo movements.

Many previous studies have reported that an increase in brainwave activity after exercise is associated with an increase in cortical activity. Whereas exercise increases the blood flow in the brain, changes in brainwaves caused by exercise are affected by diverse causes. Although changes in alpha waves after exercise were mostly interpreted as a condition during the stable state, there are also reports indicating that the activity increases at the levels of intensity ranging from low intensity to moderate intensity, that is, below the integrated threshold [28]. On the other hand, Beyer \& Schumann [29] reported that alpha activity increased less in the frontal lobe of the brain after high intensity exercise. In addition, an increase in the activity of alpha waves after exercise indicates a decrease in cortical activity and reflects a decrease in fatigue, relaxation, or a decrease in desire [30]. In this study, alpha waves showed lower activity during the kendo movements than during the resting state, and this result is considered attributable to the fact that although physical activities were not intensive during the kendo movements, the high level of tension decreased the activity of alpha waves as with highly intensive exercise. It was also consistent with reports from previous studies [31] indicating that increased visual attention resulted in a significant decrease in alpha waves.

In this study, unlike alpha waves in the low frequency region, SMR and beta waves (M-beta and $\mathrm{H}$-beta) in the high frequency region showed higher activity during kendo movements than during the resting state. Arousal and hypoxia reduce cerebral blood flow and beta-wave activity. On the other hand, when the cerebral blood flow has increased due to exercise, the brainwaves are reversed. The cerebral cortical system generally responds to an increase in metabolic arousal [32]. The increased metabolic rate and the hypothalamic regulation of body temperature during exercise affect brainwave activity, especially the activity of beta waves [33]. Exercise increases cortical activity, thereby affecting the psychological variables such as interest and arousal, and information processing processes. Therefore, an increase in the activity of the circulatory system is associated with an increase in metabolic demand and a high level of arousal due to exercise induces changes in brainwaves, and the beta wave activity increase after exercise [34].

Since violent collisions and tense confrontations with the opponent are repeated during kendo matches, a strong fighting spirit, human talent, and concentration greatly affect the outcomes of the matches. Kendo is an event in which even if an athlete has high levels of technical skills and physical strength, he/she cannot exert the ability if he/she is not mentally trained. An athlete can win the match only when he/she can make accurate judgments in the fighting with the opponent, which changes rapidly from moment to moment, and can show bold determination at each important point [15]. Therefore, in this study, it can be suggested that mental arousal was achieved due to anxiety and tension on the opponent leading to increases in body temperature and breathing so that the physical metabolic rate increased and the alpha wave activity in the low frequency region decreased but the brainwave in the high frequency region increased. However, in this study, there were a few any significant differences according to the movement of kendo. This is thought to be because there was no difference in exercise intensity because the kendo movement used in this study was composed of basic movements.

With regard to the asymmetry of the brainwaves between the left and right sides, Woo et al. [35] reported that although the brainwaves in the low-frequency region (delta, theta, alpha waves) on the left side were shown to be lower than those on the right side, the degree of the asymmetry of the brainwaves between the left and right sides increased after exercise compared to that during the resting state. In this study too, the brainwave activities on the left side of the temporal lobe and the occipital lobe were shown to be significantly lower than those on the right side after the chudan-no-kamae and three striking movements. CI on the left side of temporal lobe and occipital lobe were also shown to be lower than those on the right side. These changes are reported to be related to the duration of exercise. It has been reported that whereas exercise for a moderate duration of about 30 minutes elicits approach- 
oriented responses and suppresses withdrawal-oriented responses, exercise for more than 45-minutes suppresses approach-related responses and promotes withdrawal related responses so that the levels of brain activity on the left and right sides are reversed compared to those after exercise for 30 minutes [35]. The brain activity on the left side was shown to be lower than that on the right side in this study because the duration of exercise was relatively short at 15 minutes.

Although the mechanism of changes in brainwave activity according to exercise as such is still unclear, Craig [25] suggested it as the lateralization of the autonomic centripetality or differences in feedback to the anterior insular cortex. That is, whereas the left anterior insula is stimulated by the parasympathetic nerve, the right anterior insula is predominantly stimulated by the afferent sympathetic nervous system. The chudan-no-kamae and three striking kendo movements used in this study increased tension and concentration on the opponent's attacks but did not involve many movements and led to continuous confrontations with the opponent while controlling one's tension rather than physical excitement. Therefore, it is thought that the stimulation by the afferent sympathetic nervous system acted more than the stimulation of the parasympathetic nerve.

In addition, it is reported that an increase in brainwave activity in the frontal lobe region of the right side is associated with negative emotions [36,37], and an increase in brainwave activity in the frontal lobe region of the left side is related to motivation and self-achievement related to approaches [38]. It has been reported that in a stress situation related to withdrawal, alpha waves were shown to have an inverse relationship with brainwave activity, and an increase in alpha wave activity on the right side were shown to be related to a decrease in the right cortical activity [39]. In this study, the decrease in brainwave activity on the left side after kendo movements appeared in the temporal lobe and that occipital lobe instead of the frontal lobe suggested in previous studies. Although the cause cannot be clearly presented, it is considered attributable to the fact that brainwaves in the temporal lobe and the occipital lobe were activated due to the characteristics of the kendo movements, which are responding to the opponent while staring at the opponent, and the activity after such movements was shown to be lower on the left side than on the right side because anxiety and the level of tension on the opponent occurred rather than withdrawal.

\section{Conclusions}

The results of this study in which the activity of alpha waves during the resting state was shown to be higher than that during kendo movements (three striking movements,chudanno-kamae movement) while the activity of SMR, M-beta, and $\mathrm{H}$-beta waves during kendo movements was shown to be higher than that during the resting state are considered attributable to the fact that their concentration was partly caused by kendo training with increased anxiety and the level of tension of elementary school students thereby increasing their brainwave activity. The asymmetry between the left and right sides increased during movements compared to during the resting state and increased in the temporal lobe and the occipital lobe rather than the frontal lobe. This is considered attributable to the characteristics of kendo training in which the trainee competes with the opponent. Therefore, these results are considered as indicating that kendo training helps elementary school students increase brainwave activity through physical activities. However, the relationship between brainwaves and concentration was unclear, since CI only partly increased. It is thought that study should be conducted with the measurement of factors related to emotional aspects, concentration, and brainwaves according to kendo training.

\section{Acknowledgments}

The authors are grateful to the elementary school student who participated in this study and to the $\mathrm{C}$ city kendo coach who assisted in the experiment.

\section{Conflicts of Interest}

The authors declare that there is no conflict of interests regarding the publication of this article. 


\section{References}

1. Dik MG, Deeg DJ, Visser M, Jonker C. Early life physical activity and cognition at old age. Neuropsychol. 2003; 25(5):643-653.

2. Brismar T. The human EEG physiological and clinical studies. Physiology Behavior. 2007; 10:92(1-2):141-147.

3. Petruzzello SJ, Landers DM, Hatfield BD, Kubitz KA, Salazar W. A meta-analysis on the anxiety-reducing effects of acute and chronic exercise. Outcome Med Sport Med. 1991; 11:143-182.

4. Boutcher SH. Emotion and aerobic exercise. In: Singer RN, Murphey M, Tennant LK, editors. Handbook of Res Sport. Manag Psychol. New York: MacMillan. 2014; 799-814.

5. Sterman MB. Sensorimotor EEG operant conditioning and experimental and clinical effects. Pavlovian J Biol Sci. 1977; 12(2):65-92.

6. Lubar JF. Discourse on the development of EEG diagnostics and biofeedback for attention-defit hyperactivity disorders. Biofeedback Self-Regul. 1991; 16:201-225.

7. Brain Science Institute. Go to brain utilization test: Smart Brain Manual. Brain Science Research Institute Press. 2014. p 100-130.

8. Kober SE, Witte M, Stangl M, Väljamäe A, Neuper C, Wood G. Shutting down sensorimotor interference unblocks the networks for stimulus processing: an SMR neurofeedback training study. Clin Neurophysio. 2015; 126(1):82-95.

9. Moraes H, Deslandes A, Silveira H, Ribeiro P, Cagy M, Piedade R, Pompeu F, Laks J. The effect of acute effort on EEG in healthy young and elderly subjects. Eur J Appl Physiol. 2011; 111(1):67-75.

10. Kubitz KA, Mott AA. EEG Power Spectral Densities During and After Cycle Ergometer Exercise. Res Q Exerc Sport. 1996; 67(1):91-96.

11. Hillman CH, Pontifex MB, Raine LB, Castelli DM, Hall EE, Kramer AF. The effect of acute treadmill walking on cognitive control and academic achievement in preadolescent children. Neurosci. 2009; 159(3):10441054.
12. Colcombe SJ, Erickson KI, Raz N, Webb AG, Cohen NJ, McAuley E, Kramer AF. Aerobic fitness reduces brain tissue loss in aging humans. J Gerontol A Biol Sci Med Sci. 2003; 58(2):176-180.

13. Ko DS. Different EEG changes in image training of kumdo players. Yonsei University Graduate School Master's Thesis. 2001, p 50-70.

14. Han SH, Kim BJ, Oh BJ. A study on changes in brain wave and body rhythm of kendo players depending on whether or not they have video stimulation. KCDC 2014; 16(4):163-170.

15. Park SS, Park JJ. Analysis of Determinants of Kendo Performance Using Delphi Technique. KMA. 2017; 19(2):137-148.

16. Treewiki. Brain Science Project. https://namu.wiki. (Accessed Sep 10, 2020)

17. Sterman MB. Basic concepts and clinical findings in the treatment of seizure disorders with EEG operant conditioning. J Clin Electrophysiol. 2000; 31(1):45-55.

18. Lee KH, Ru YS, Moon SH. Improvement of children's attentiveness through robot educational activities. Korean Society for Practical Education. 2010; 185-204.

19. Lee JE, Park SM. The effect of sound wave application training on the concentration of kendo athletes. KMA. 2017; 19(3):33-45.

20. Allen JJ, Harmon-Jones E, Cavender JH. Manipulation of frontal EEG asymmetry through biofeedback alters self-reported emotional responses and facial EMG. Psychophysiol. 2001; 38:685-693.

21. Hall EE, Ekkekakis P, Petruzzello SJ. Regional brain activity and strenuous exercise: Predicting affective responses using EEG asymmetry. Biol Psychol. 2007; 75:194-200

22. Petruzzello SJ, Landers DM. State anxiety reduction and exercise: does hemispheric activation reflect such changes? Med Sci Sport Exerc. 1994; 26:1028-1035.

23. Petruzzello SJ, Tate AK. Brain activation, affect and aerobic exercise: an examination of both state-independent and state-dependent relationships. Psychophysiol. 1997; 34:527-533.

24. Petruzzello SJ, Hall EE, Ekkekakis P. Regional brain activation as a biological marker of responsivity to 
acute exercise: influence of fitness. Psychophysiol. 2001; 38:99-106.

25. Craig AD. Forebrain emotional asymmetry: a neuroanatomical basis? Trends Cogn Sci. 2005; 9:566571.

26. Gasser T, Bacher P, Mocks J. Transformations towards the normal distribution of broad band spectral parameters of the EEG Electroencephalogram. Clin Neurophysiol. 1982; 53:119-124.

27. Kang SH, Park JH, Shin SW, Chung ST. Analysis of EEG signals for attention training game contents. IIBC. 2019; 19(3):83-90.

28. Bailey S, Hall E, Cain J, Miller P, Folger S. Changes in the brain activity during a graded exercise test on a renumber cycle ergometer. Med Sci Sport Exerc. 2004; 36:S286.

29. Beyer L, Schumann H. Möglichkeitenneuro physiologischer Untersuchungen in der Sportmedizinanhand von zweiausgew Ählten Beispielen. MED Sport. 1981; 3:65-70

30. Crabbe JB, Dishman RK. Brain electrocortical activity during and after exercise: a quantitative synthesis. Psychophysiol. 2004; 41:563-574.

31. Weinstein S, Valentine A, Weinstein C. Brain-Activity Responses to Magazine and Television Advertising. J Advert Res. 1980; 20(3):59-63.

32. Kamijo K, Nishihira Y, Hatta A, Kaneda T, Kida T, Higashiura T, Kuroiwa K. Changes in arousal level by differential exercise intensity. Clin Neurophysiol. 2004; 115:2693-2698.

33. Nielsen B, Nybo L. Cerebral changes during exercise in the heat. Sports Med. 2003; 33:1-11.

34. Ogoh S, Fadel PJ, Zhang R, Selmer C, Jans Q, Secher NH, Raven PB. Middle cerebral artery flow velocity and pulse pressure during dynamic exercise in humans. AJP- Heart and Circ. 2005; 288:1526-1531. 21.

35. Woo M, Kim S, Kim J, Petruzzello SJ, Hatfield BD. Examining the exercise-affect dose-response relationship: does duration influence frontal EEG asymmetry? Int J Psychophysiol. 2009; 72(2):166-72.

36. Allen JJ, Kline JP. Frontal EEG asymmetry, emotion, and psychopathology: the first, and the next 25 years. Biol Psychol. 2004; 67(1-2):1-5.

37. Schutter DJ, Weijer AD, Meuwese JD, Morgan B, Honk JV. Interrelations between motivational stance, cortical excitability, and the frontal electroencephalogram asymmetry of emotion: a transcranial magnetic stimulation study. Hum Brain Mapp. 2008; 29(5):574580.

38. Coan JA, Allen JJB. Frontal EEG as a moderator and mediator of emotion. Biol Psychol. 2004; 67:7-49.

39. Cooper NR, Croft RJ, Dominey SJ, Burgess AP, Gruzelier JH. Paradox lost? Exploring the role of alpha oscillations during externally vs. internally directed attention and the implications for idling and inhibition hypotheses. Int J Psychophysiol. 2003; 47(1):65-74. 